\title{
Aberrant expression of SPAG6 may affect the disease phenotype and serve as a tumor biomarker in BCR/ABL1-negative myeloproliferative neoplasms
}

\author{
LI DING $^{1,2^{*}}$, JIE LUO $^{1 *}$, JING PING ZHANG $^{2}$, JI WANG $^{2}$, ZHAO QUAN LI ${ }^{1}$, JUAN HUANG $^{3}$, LI CHAI $^{3}$, \\ JIAO MU ${ }^{1}$, BEIBEI ZHAO ${ }^{1}$, YI RUI ZHONG ${ }^{1}$, LIN YI ZHANG ${ }^{1}$ and LIN LIU ${ }^{1}$ \\ ${ }^{1}$ Department of Hematology, The First Affiliated Hospital of Chongqing Medical University, Chongqing 400042; \\ Departments of ${ }^{2}$ Hematology and ${ }^{3}$ Pathology, The Affiliated Hospital of Southwest Medical University, \\ Luzhou, Sichuan 646000, P.R. China
}

Received July 22, 2021; Accepted October 15, 2021

DOI: 10.3892/ol.2021.13128

\begin{abstract}
Sperm-associated antigen 6 (SPAG6) is a newly identified cancer-testis antigen that has been revealed to contribute to the occurrence and development of various types of human cancer, such as ovarian, bladder, breast and lung cancer. However, to the best of our knowledge, the expression levels of SPAG6 in breakpoint cluster region (BCR)/ABL1-negative myeloproliferative neoplasms (MPNs) have not been investigated previously. Using reverse transcription-quantitative PCR and different tissue staining techniques, the present study revealed that SPAG6 was expressed by MPN cells, both at the mRNA and protein levels, and that nucleated erythroid precursors and megakaryocytes expressed the highest levels of SPAG6. In addition, SPAG6, which is known as a microtubule-associated protein, was found to exhibit nucleic, cytoplasmic or both cytoplasmic and nucleic subcellular localization patterns within the same patient or cell type; however, it did not always co-localize with $\beta$-tubulin. Furthermore, SPAG6 expression was revealed to be associated with fewer splenomegaly $[\mathrm{P}=0.015$ for polycythemia vera $(\mathrm{PV})$ and essential thrombocythemia $(\mathrm{ET})$; and $\mathrm{P}=0.012$ for primary myelofibrosis (PMF)] and myelofibrosis events $(\mathrm{P}=0.014$ for $\mathrm{PV}$ and ET; and $\mathrm{P}=0.004$ for $\mathrm{PMF}$ ). In patients with $\mathrm{PMF}$, upregulated expression levels of SPAG6 were also found to be associated with lower white blood cell counts $(\mathrm{P}=0.042)$ and lactate dehydrogenase levels $(\mathrm{P}=0.012)$, and higher hemoglobin levels $(\mathrm{P}=0.031)$ and platelet counts $(\mathrm{P}=0.025)$. In addition, the
\end{abstract}

Correspondence to: Dr Lin Liu, Department of Hematology, The First Affiliated Hospital of Chongqing Medical University, 1 Youyi Road, Chongqing 400042, P.R. China

E-mail: liulin@cqmu.edu.cn

*Contributed equally

Key words: sperm-associated antigen 6, aberrant expression, myeloproliferative neoplasms, disease phenotype, tumor biomarker receiver operating characteristic curve analysis indicated that SPAG6 may be a potential biomarker for distinguishing MPN cases from healthy individuals. In conclusion, to the best of our knowledge, the present study is the first to report that aberrant SPAG6 expression may affect the disease phenotype and serve as a tumor biomarker in BCR/ABL1-negative MPNs.

\section{Introduction}

Breakpoint cluster region (BCR)/ABL1-negative myeloproliferative neoplasms (MPNs) are a group of clonal hematological malignancies comprising polycythemia vera (PV), essential thrombocythemia (ET) and primary myelofibrosis (PMF) (1). Clinical manifestations of MPNs include the excessive production of one or more fully differentiated blood cell types, constitutional symptoms, splenomegaly and a propensity for thrombosis or excessive bleeding (2). In addition, ET and PV can progress to MF, and all types of MPN may eventually progress to more severe bone marrow hematopoietic failure or acute myeloid leukemia (AML) (2). Thrombosis, bleeding and disease progression are the most serious complications of MPNs, and often the most common causes of mortality in patients (3).

MPN subtypes, including ET, PV and PMF, are often referred to together, as they have similar pathobiological and clinical features (2). Although patients share similar driver gene mutations in Janus kinase (JAK)2 (4), MPL proto-oncogene thrombopoietin receptor (MPL) (5) and calreticulin (CALR) (6), which are responsible for the constitutive activation of the JAK/STAT signaling pathway, there are a number of unknown cooperating molecular and genetic aberrations that contribute to the disease phenotypes, transformation and progression (7). Current treatment regimens involve cytoreductive therapy, aspirin and novel drugs targeting specific gene mutations, such as JAK inhibitors, which successfully control symptoms and reduce spleen volume, but do not necessarily halt disease progression $(2,8)$. Allogeneic stem cell transplantation remains the only curative therapy option available for patients with MPNs; however, it is only offered to a limited number of patients with MF with advanced-risk 
disease and without prohibitive comorbidities (9). Therefore, it is of great clinical significance to identify novel biomarkers to predict the prognosis of MPNs and optimize individual treatment strategies.

Sperm-associated antigen 6 (SPAG6) was first detected in human testicular tissue and identified as a novel cancer-testis antigen (10). In mammals, SPAG6 is predominately expressed in the sperm, lungs, nervous system and inner ear, and is considered to be mainly involved in sperm maturation and nervous system development under normal physiological conditions $(11,12)$. Accumulating evidence has demonstrated that aberrant SPAG6 expression in hematological malignancies, and ovarian, bladder, breast and lung cancer, may affect the occurrence and development of human cancer types by regulating the proliferation, apoptosis, invasion and metastasis of tumor cells (13-20). In addition, SPAG6 has been reported to enhance the effect of apoptosis-inducing drugs and induce cell cycle arrest (21). These findings suggest that SPAG6 may represent a potential tumor marker and a promising antitumor therapeutic target.

To the best of our knowledge, the role of SPAG6 in MPNs has not been previously described, although aberrant SPAG6 expression has been extensively reported in other types of disease. The present study aimed to analyze the expression levels of SPAG6 in patients with MPNs and to determine the influence of SPAG6 expression on MPN clinical characteristics, which may offer insights into novel biomarkers and therapeutic targets for MPNs.

\section{Materials and methods}

Patient samples. A total of 219 patients with MPNs and 93 individuals in the control group were enrolled in the present study (Fig. 1). Bone marrow specimens and clinical data were obtained from newly diagnosed patients with MPNs and individuals in the control group who were admitted to The First Affiliated Hospital of Chongqing Medical University (Chongqing, China) between January 2018 and June 2021. The use of bone marrow specimens was approved by the Ethics Committee of The First Affiliated Hospital of Chongqing Medical University (approval no. 2021-109) and written informed consent was obtained from all participants (consent was obtained from parents/guardians for minors). The cases included in the present study met the diagnostic criteria of the 2016 World Health Organization classification and diagnostic criteria for MPNs (1). A summary of the specific research scheme is shown in Fig. 1. The main clinical and laboratory features of the patients with MPNs that had undergone a bone marrow biopsy are presented in Table I. In the present study, 'with myelofibrosis' refers to patients with MPNs whose bone marrow biopsy revealed abnormally reactive deposition of marrow stromal reticulin and collagen fibers [marrow fibrosis $(\mathrm{MF}) \geq 1$ ]. It is worth noting that PMF can be divided into pre-fibrosis (pre-PMF) and overt-fibrosis (overt-PMF) subtypes (1). For pre-PMF cases, an MF $\leq 1$ is required, thus patients with $\mathrm{PMF}$ may also have bone marrow without fibrosis (1). The age of patients that had undergone reverse transcription-quantitative PCR (RT-qPCR) ranged between 27 and 80 years for MPNs, and 19 and 78 years for the control groups. The sex distribution of these patients was split
$57.6 \%$ male and $42.4 \%$ female for MPNs, and $55.0 \%$ male and $45.0 \%$ female for the control groups. Since these patients had different diseases, clinical data were not compared between these groups.

$R T$-qPCR. Total bone marrow nucleated cellular RNA was isolated from each sample using TRIzol ${ }^{\circledR}$ (Total RNA Isolation) reagent (Invitrogen; Thermo Fisher Scientific, Inc.). Total RNA was reverse transcribed into cDNA using a reverse transcription kit (PrimeScript ${ }^{\mathrm{TM}}$ RT reagent Kit; Takara Biotechnology Co., Ltd.) according to the manufacturer's instructions. qPCR was subsequently performed using a CFX96 Real Time PCR Detection system (Bio-Rad Laboratories, Inc.). The PCR system and cycle parameters used were as previously described (22). The total reaction volume was $10 \mu \mathrm{l}$ and this was prepared as follows: $5 \mu \mathrm{l}$ TB Green Master Mix (Takara Biotechnology Co., Ltd.),

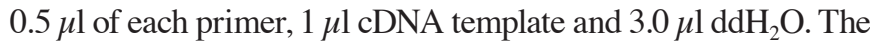
thermocycling conditions used for qPCR were as follows: $95^{\circ} \mathrm{C}$ for $30 \mathrm{sec}$, followed by 40 cycles at $95^{\circ} \mathrm{C}$ for $5 \mathrm{sec}$ and $60^{\circ} \mathrm{C}$ for $30 \mathrm{sec}$. The relative mRNA expression levels of SPAG6 were calculated using the $2^{-\Delta \Delta \mathrm{Cq}}$ method (23), and GAPDH acted as the internal control. The following primers sequences were used for qPCR: SPAG6 forward, 5'-AGTGCGACATTCTTCCACAGC TTG-3' and reverse, 5'-GCG TATCCAGTGCTCCACAATCG-3'; and GAPDH forward, 5'-CTTTGGTATCGTGGAAGGACTC-3' and reverse, 5'-GTAGAGGCAGGGATGATGTTCT-3'.

\section{Bone marrow biopsy specimen staining techniques}

Immunohistochemistry. Tissues were fixed with $3 \%$ neutral formaldehyde fixative at room temperature for $>24 \mathrm{~h}$, decalcified, routinely processed for paraffin embedding and then cut into $3-\mu \mathrm{m}$-thick sections for staining. The slides were subsequently deparaffinized using xylene (xylene I for $15 \mathrm{~min}$; xylene II for $15 \mathrm{~min}$; xylene III for $15 \mathrm{~min}$ ) and rehydrated using ethanol (absolute ethanol I for $5 \mathrm{~min}$; absolute ethanol II for $5 \mathrm{~min}$; $85 \%$ alcohol for $5 \mathrm{~min}$; $75 \%$ alcohol for $5 \mathrm{~min}$ ) after being dried at $60^{\circ} \mathrm{C}$ for $30 \mathrm{~min}$. The sections were incubated with sodium citrate $\left(0.1 \mathrm{mM}\right.$; pH 6.0) for $30 \mathrm{~min}$ at $98^{\circ} \mathrm{C}$ for antigen retrieval and then incubated with $3 \% \mathrm{H}_{2} \mathrm{O}_{2}$ at room temperature in the dark for $25 \mathrm{~min}$ to eliminate the endogenous peroxidase activity. Subsequently, 3\% BSA (cat. no. G5001; Wuhan Servicebio Technology Co., Ltd.) was added to cover the marked tissue to block non-specific binding at room temperature for $30 \mathrm{~min}$. Following these incubations, the slides were incubated with a rabbit anti-SPAG6 antibody (dilution, 1:400; cat. no. Bs-12291R; BIOSS) overnight at $4^{\circ} \mathrm{C}$. After the primary antibody incubation, the sections were incubated with a horseradish peroxidase-conjugated goat anti-rabbit secondary antibody (dilution, 1:200; cat. no. GB-23303; Wuhan Servicebio Technology Co., Ltd.) at room temperature for $50 \mathrm{~min}$, and were then incubated with 3,3'-diaminobenzidine (DAB), as the chromogen, using the DAB Detection kit (cat. no. G1211; Wuhan Servicebio Technology Co., Ltd.), prior to being counterstained with hematoxylin at room temperature for $\sim 3 \mathrm{~min}$. Each section incubated with the anti-SPAG6 antibody was compared with an adjacent section stained with rabbit serum (cat. no. G1209; Wuhan Servicebio Technology Co., Ltd.) as the negative control. All stained sections were visualized using an Imager A2 microscope (light microscope; Zeiss $\mathrm{GmbH}$ ). 
Table I. Major clinical characteristics of patients with myeloproliferative neoplasms.

\begin{tabular}{|c|c|c|c|c|}
\hline Variable & $\mathrm{PV}(\mathrm{n}=51)$ & $\mathrm{ET}(\mathrm{n}=91)$ & $\operatorname{PMF}(\mathrm{n}=31)$ & P-value \\
\hline Sex (male/female), n (\%) & $28 / 23(54.9 / 45.1)$ & $44 / 47(48.4 / 51.6)$ & $17 / 14(54.8 / 45.2)$ & 0.692 \\
\hline Median age at onset (range), years & $61(31-75)$ & $53(15-88)$ & $67(42-83)$ & $0.008^{\mathrm{a}, \mathrm{b}}$ \\
\hline Median WBC count (range), x $10^{9} /$ liter & $12.03(4.05-31.34)$ & $10.11(4.32-31.00)$ & $15.61(1.97-70.89)$ & $0.005^{\mathrm{a}, \mathrm{b}}$ \\
\hline Median hemoglobin (range), g/liter & $192(161-245)$ & $132(108-158)$ & $99(44-121)$ & $<0.001^{\mathrm{a}-\mathrm{c}}$ \\
\hline Median PLT count (range), x109/liter & $354(110-1182)$ & $958(457-2517)$ & $248(7-1056)$ & $<0.001^{\mathrm{b}, \mathrm{c}}$ \\
\hline Median LDH (range), U/liter & $285.6(18.7-685.0)$ & $243.2(45.8-694.2)$ & $407.1(68.7-1011.2)$ & $<0.001^{\mathrm{a}, \mathrm{b}}$ \\
\hline Gene mutation, n (\%) & $51(100.0)$ & $66(72.5)$ & $27(87.1)$ & $<0.001^{\mathrm{a}, \mathrm{c}}$ \\
\hline JAK2 & $51(100.0)$ & $45(49.4)$ & $20(64.5)$ & \\
\hline CALR & $0(0.0)$ & $17(18.7)$ & $5(16.1)$ & \\
\hline MPL & $0(0.0)$ & $4(4.4)$ & $2(6.5)$ & \\
\hline Triple-negative & $0(0.0)$ & $25(27.5)$ & $4(12.9)$ & \\
\hline Median H-score (range) & $2.55(0.80-3.56)$ & $2.60(0.12-3.72)$ & $1.42(0.08-3.48)$ & $<0.001^{\mathrm{a}, \mathrm{b}}$ \\
\hline
\end{tabular}

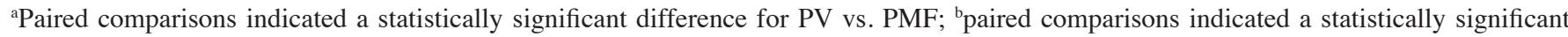
difference for ET vs. PMF; c paired comparisons indicated a statistically significant difference for PV vs. ET. CALR, calreticulin; ET, essential thrombocythemia; JAK2, Janus kinase 2; LDH, lactate dehydrogenase; MPL, MPL proto-oncogene, thrombopoietin receptor; PLT, platelet; PMF, primary myelofibrosis; PV, polycythemia vera; WBC, white blood cell.

Pathological analysis was performed independently by two experienced pathologists at the Pathology Department of The Affiliated Hospital of Southwest Medical University (Luzhou, China) who were blinded to the final clinical diagnosis of all cases studied. The immunoreactivity of SPAG6 in the bone marrow biopsy samples was semi-quantified using the McCarty's H-score system (24), which incorporates both the intensity of the specific staining and the percentage of positive cells. For each section stained, four high-power fields (magnification, x400) were randomly selected for analysis and the $\mathrm{H}$-score was calculated using the following formula: $\Sigma \mathrm{Pi}(\mathrm{I}+1)$, whereby I represented the relative intensity of specific staining (no staining, 0 ; weak but detectable above control levels, 1+; distinct, 2+; and very strong, 3+) and Pi represented the percentage of positive cells. The final score was the sum of the relative intensity of specific staining multiplied by the percentage of positive cells.

$H \& E$ staining. Tissues were fixed with $3 \%$ neutral formaldehyde fixative at room temperature for $>24 \mathrm{~h}$, decalcified, routinely processed for paraffin embedding and then cut into $3-\mu$ m-thick sections for staining. The slides used for $\mathrm{H} \& \mathrm{E}$ staining were first deparaffinized and rehydrated as aforementioned. The sections were then stained with hematoxylin (cat. no. G1004; Wuhan Servicebio Technology Co., Ltd.) at room temperature for $2 \mathrm{~min}$, rinsed with running water for $10 \mathrm{sec}$ and differentiated using $1 \%$ hydrochloric acid-ethanol for $10 \mathrm{sec}$. After washing with distilled water for $1 \mathrm{~min}$, the sections were stained with $0.5 \%$ eosin solution (cat. no. G1002; Wuhan Servicebio Technology Co., Ltd.) at room temperature for $1 \mathrm{~min}$, washed in distilled water for $10 \mathrm{sec}$, dehydrated using a gradient series of ethanol, cleared using xylene and sealed with neutral balsam. Stained cells were visualized using an Imager A2 microscope (light microscope; Zeiss $\mathrm{GmbH}$ ).

Immunofluorescence staining. Tissue sections underwent double immunofluorescence staining. Briefly, staining for the first antibody was performed according to the immunohistochemical procedure. The sections were incubated with 3\% BSA (cat. no. G5001; Wuhan Servicebio Technology Co., Ltd.) to block non-specific binding at room temperature for $30 \mathrm{~min}$, and were then incubated overnight at $4^{\circ} \mathrm{C}$ with the following primary antibodies: Anti-SPAG6 (rabbit; dilution, 1:200; cat. no. Bs-12291R; BIOSS) and anti- $\beta$-tubulin (mouse; dilution, 1:200; cat. no. GB13433; Wuhan Servicebio Technology Co., Ltd.). Following the primary antibody incubation, the samples were incubated with an appropriate fluorophore-conjugated secondary antibody (goat anti-rabbit/goat anti-mouse; dilution, 1:400/1:300; cat. no. GB25303/GB21301; Wuhan Servicebio Technology Co., Ltd.) at room temperature for $50 \mathrm{~min}$ in the dark. The nuclei were stained with DAPI (cat. no. G1401; Wuhan Servicebio Technology Co., Ltd.) at room temperature for $10 \mathrm{~min}$ in the dark. and the staining results were observed using a confocal laser scanning microscope (Eclipse Ti; Nikon Corporation).

Statistical analysis. Statistical analysis was performed using SPSS (version 25.0; IBM Corp.) and GraphPad Prism (version 8.0; GraphPad Software, Inc.) software. Mann-Whitney U test or Kruskal-Wallis one-way analysis of variance followed by Bonferroni's post hoc test was used to compare the between-group differences of continuous variables, and Pearson's $\chi^{2} /$ Fisher's exact test was used to compare the between-group differences of categorical variables. Numerical data are presented as the median and range, while categorical data are presented as count and relative frequency (\%) of each category. The receiver operating characteristic (ROC) curve and area under the curve (AUC) were used to assess the discriminative capacity of SPAG6 expression among patients and controls. All experiments were performed in triplicate. $\mathrm{P}<0.05$ was considered to indicate a statistically significant difference. 


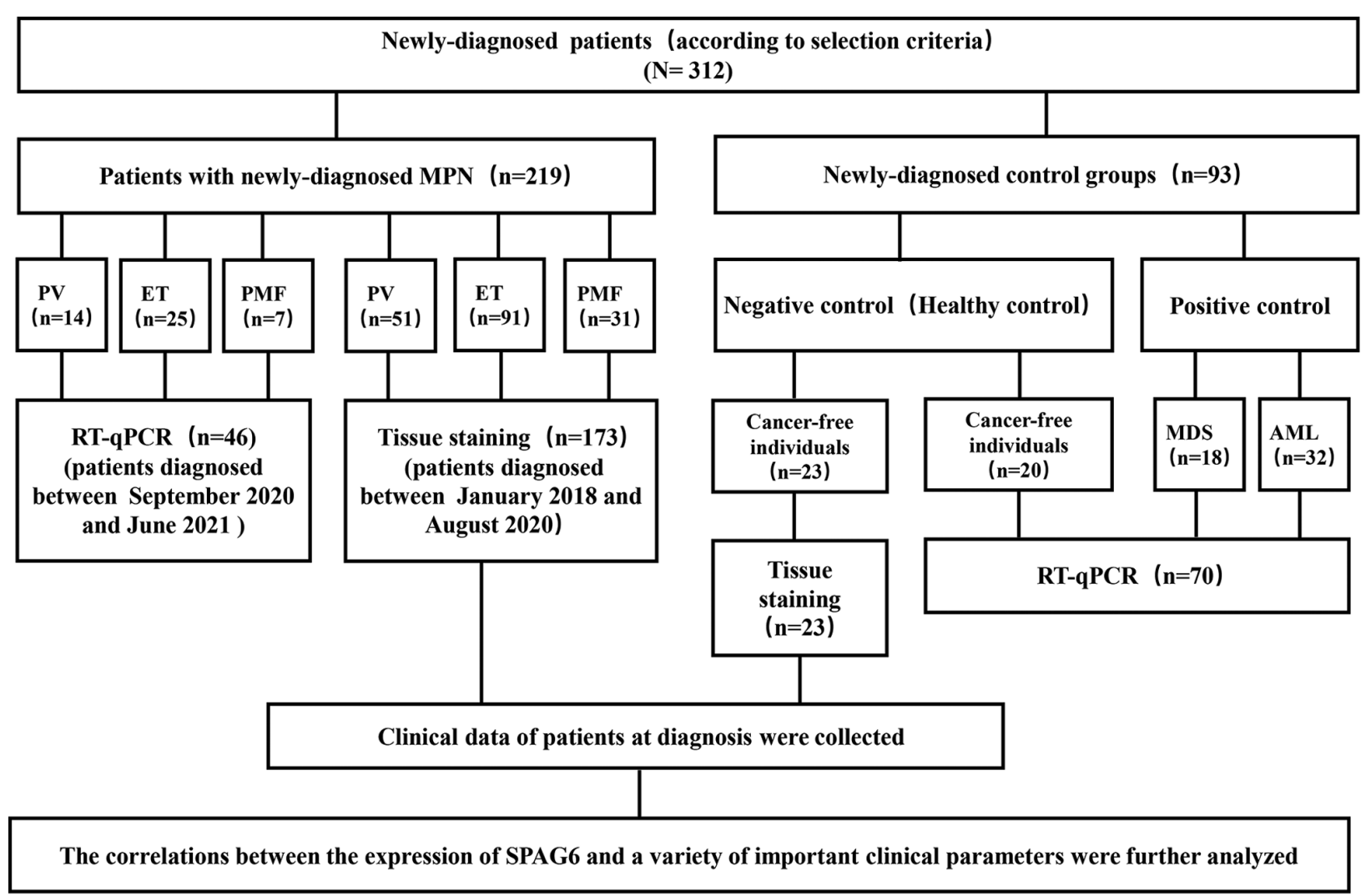

Figure 1. Study design. AML, acute myeloid leukemia; ET, essential thrombocythemia; MDS, myelodysplastic syndrome; MPN, myeloproliferative neoplasm; PMF, primary myelofibrosis; PV, polycythemia vera; RT-qPCR, reverse transcription-quantitative PCR; SPAG6, sperm-associated antigen 6; WB, western blotting.

\section{Results}

Major demographic, hematological, biochemicaland genetic mutation findings in patients with MPNs. To determine the association between the expression levels of SPAG6 and important clinical parameters in patients with MPNs, 173 MPN cases that had undergone a bone marrow biopsy were selected (Fig. 1). Cases were distributed as follows: 91 patients with ET, 51 patients with $\mathrm{PV}$ and 31 patients with PMF (including 6 pre-PMF and 25 overt-PMF cases). The clinical data of these patients were reviewed (Table I). In the present study, patients with PMF had the highest lactate dehydrogenase levels; patients with PV had the highest hemoglobin levels and gene mutation rate; patients with ET had the highest platelet counts, and the frequency of mutations was similar to that in PMF $(72.5 \%$ for ET and $87.1 \%$ for PMF). The major demographic, hematological, biochemical and genetic mutation findings in patients with MPNs were revealed to be similar to those reported in previous studies $(2,4-6)$.

Expression levels of SPAG6 in patients with MPNs. To determine whether SPAG6 expression is upregulated in patients with MPNs, RT-qPCR was performed using RNA extracted from whole bone marrow cells of 46 patients with newly diagnosed MPN and 70 control cases (Fig. 1). The results revealed that SPAG6 mRNA expression levels were significantly upregulated in patients with PV, ET and PMF compared with those in healthy controls $(\mathrm{P}<0.01$; Fig. 2Aa), and the SPAG6 mRNA expression levels in patients with MPN, including patients with
PV, ET and PMF, were similar to those of patients with MDS and AML ( $\mathrm{P}>0.05$; Fig. 2Ab).

To identify the populations of MPN bone marrow cells that expressed SPAG6, various staining techniques were performed using bone marrow biopsy samples from 173 patients and 23 healthy controls (Fig. 1). Representative immunohistochemical results of SPAG6 expression in all MPN groups are shown in Fig. 3. The immunoreactivity of SPAG6 in the bone marrow biopsy samples was semi-quantified using McCarty's H-score system and the results are shown in Fig. 2B and Table I. The $\mathrm{H}$-scores of SPAG6 immunoreactivity in the PV, ET and PMF groups were significantly increased compared with those in the control group $(\mathrm{P}<0.01$; Fig. $2 \mathrm{~B})$. The $\mathrm{H}$-score of SPAG6 immunoreactivity in the PMF group was significantly decreased compared with that of the PV and ET groups $(\mathrm{P}<0.01)$; however, the $\mathrm{H}$-score of SPAG6 immunoreactivity in the PV group was not significantly different compared with that of the ET group $(\mathrm{P}>0.05$; Table I).

Cell populations expressing SPAG6 in patients with MPNs. It was found that the cell populations expressing SPAG6 were heterogeneous among all patients with MPNs (Fig. 4). However, positive immunoreactivity of SPAG6 was mainly observed in nucleated erythroid precursors $(n=149)$ and megakaryocytes $(n=135)$. For the vast majority of patients, these cells had the highest levels of SPAG6 expression, suggesting that they were a predominate source of SPAG6 expression. In addition, positive immunoreactivity of SPAG6 was also observed in lymphocytes $(n=127)$, osteocytes $(n=115)$, osteogenic cells $(n=106)$, osteoblasts $(n=103)$, marrow stroma cells $(n=113)$ and endothelial 
A

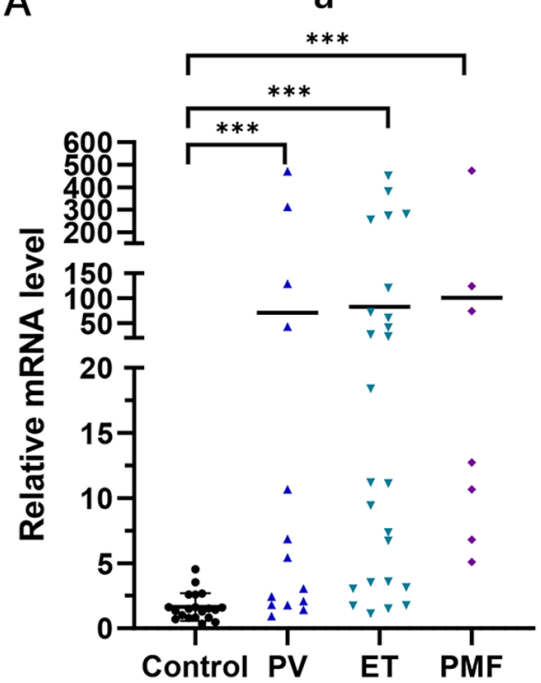

b

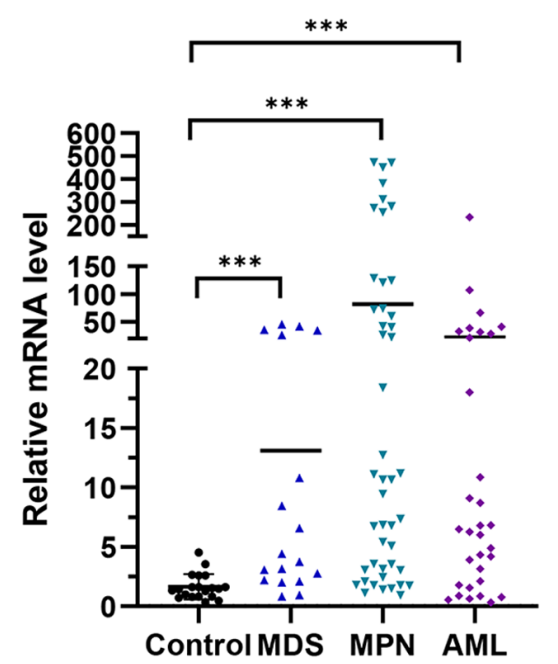

B

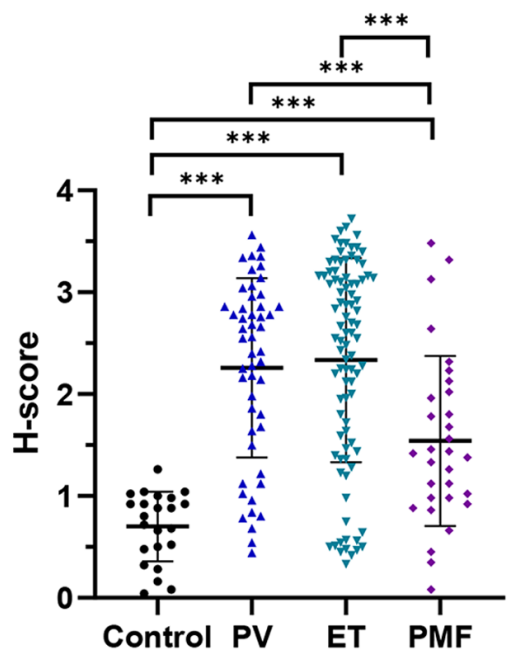

Figure 2. Relative expression levels and immunoreactivity H-score of SPAG6. (A) SPAG6 mRNA expression in (a) PV, ET, PMF and healthy control groups, and (b) MPN, MDS, AML and healthy control groups. (B) H-score of SPAG6 immunoreactivity in PV, ET, PMF and healthy control groups. ${ }^{* * * *} \mathrm{P}<0.01$ AML, acute myeloid leukemia; ET, essential thrombocythemia; MDS, myelodysplastic syndrome; MPN, myeloproliferative neoplasm; PMF, primary myelofibrosis; PV, polycythemia vera; SPAG6, sperm-associated antigen 6.
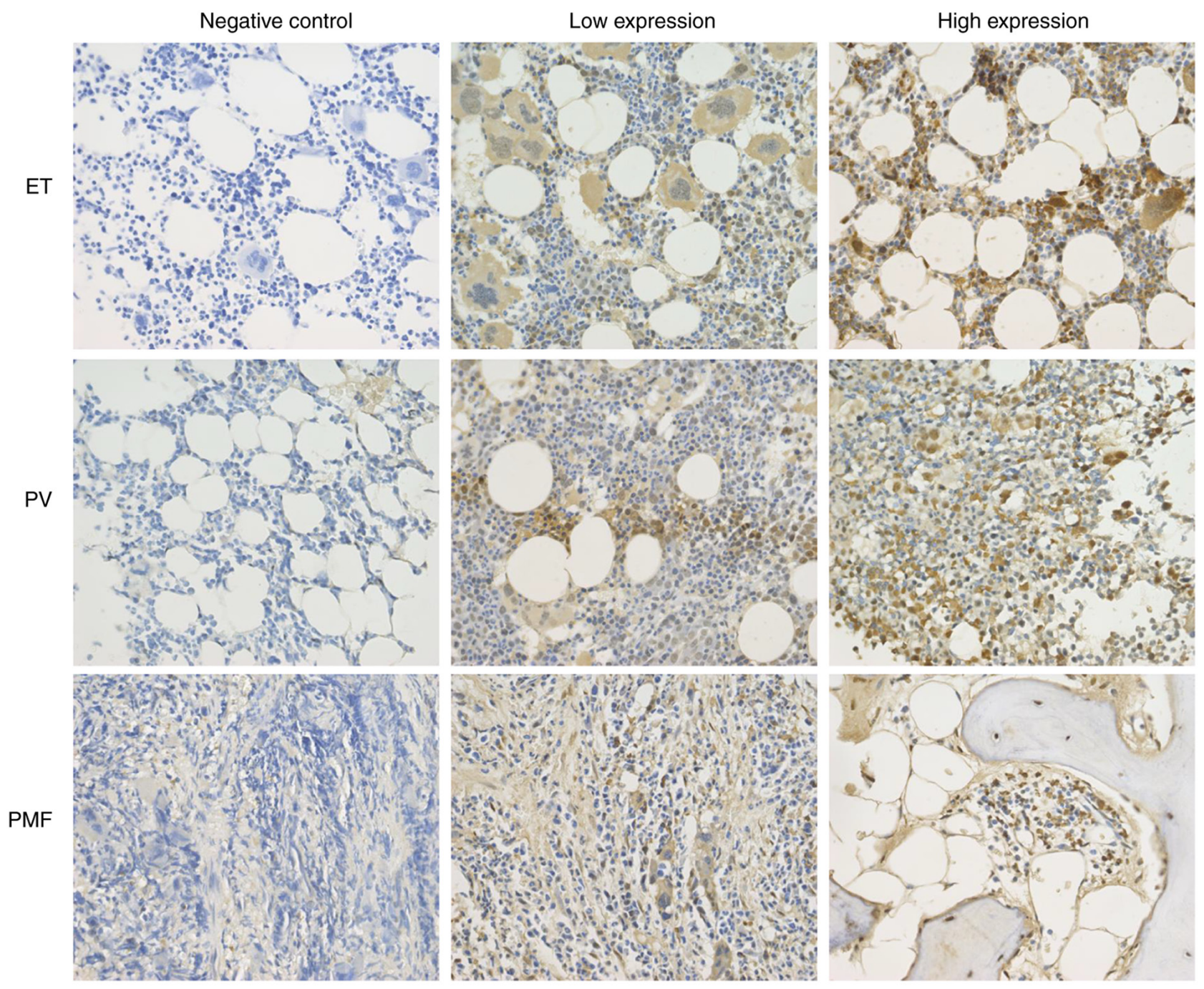

Figure 3. Representative immunohistochemical findings in all myeloproliferative neoplasm groups. Immunohistochemical analysis of sperm-associated antigen 6 expression was performed using paraffin-embedded bone marrow biopsy specimens. ET (upper panels), PV (middle panels) and PMF (lower panels). Negative control (left panels), low expression (center panels) and high expression (right panels). Magnification, $\mathrm{x} 400$. ET, essential thrombocythemia; PMF, primary myelofibrosis; PV, polycythemia vera. 


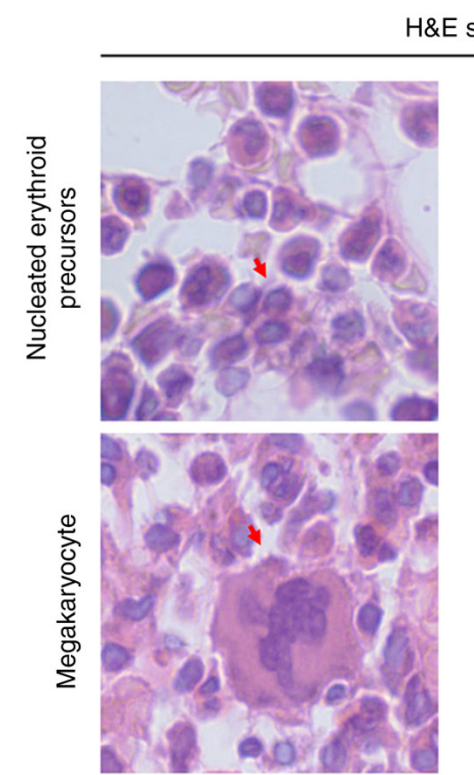

E staining
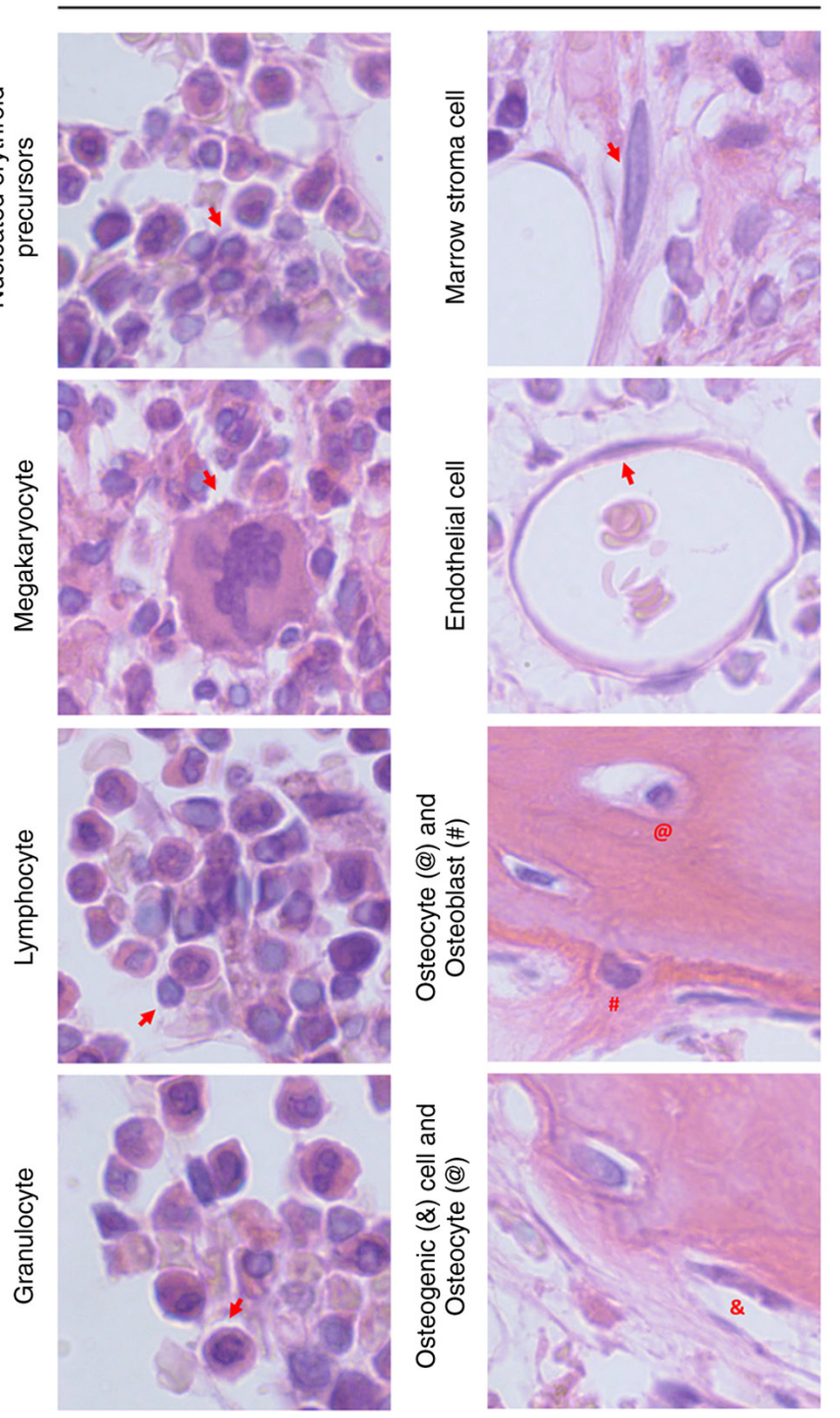
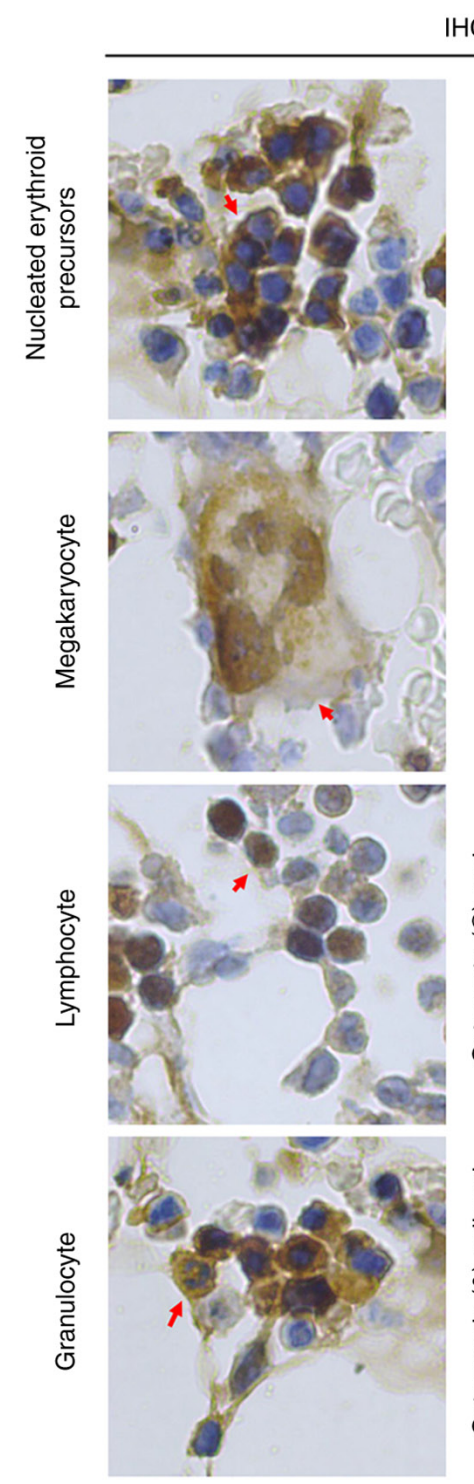

IHC staining
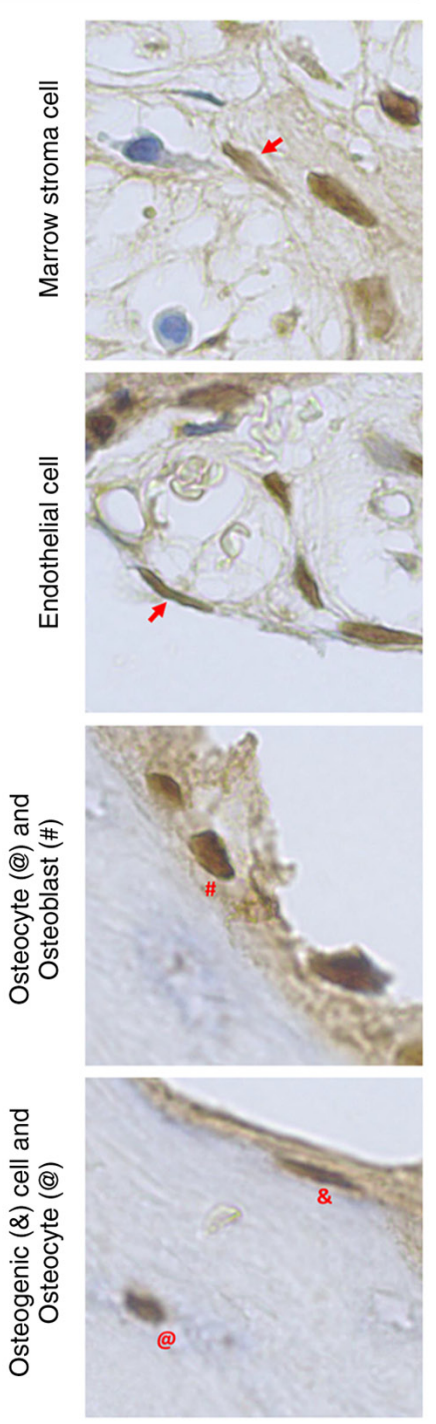

Figure 4. Cell populations expressing SPAG6 in patients with myeloproliferative neoplasms. Sections were stained using H\&E staining (left panels) and SPAG6 IHC staining (right panels). Original magnification, x1,000. The arrows point to the cell types indicated on the left. @, osteocyte; \#, osteoblast; and $\&$, osteogenic. IHC, immunohistochemistry; SPAG6, sperm-associated antigen 6.

cells $(n=105)$; however, these cells only account for a small proportion of cells present in the bone marrow, and thus, their overall contribution was limited. Notably, only a few granulocytes in 3 cases were found to express SPAG6 (data not shown).

Subcellular localization patterns of SPAG6 protein in patients with MPNs. To further clarify whether SPAG6 was involved in the regulation of the microtubule/cytoskeletal system in MPNs, tissue sections underwent double immunofluorescence staining. The immunohistochemical and immunofluorescence findings revealed that SPAG6, which is known to be a microtubule-associated protein (20), exhibited nucleic, cytoplasmic or both cytoplasmic and nucleic subcellular localization patterns in the same patient (Fig. 5A) or cell type (Fig. 5B). In addition, the results of the double immunofluorescence staining demonstrated that the SPAG6 protein did not always co-localize with $\beta$-tubulin (Fig. 6), indicating that SPAG6 may also exert other functions in addition to binding with $\beta$-tubulin.
Association of SPAG6 expression with clinical characteristics in MPNs. It has been reported that PV and ET share similar pathobiological and clinical features, while PMF presents with different clinical characteristics (2). Therefore, in the present study, results from PV and ET cases were referred to together, while results associated with PMF cases were referred to separately. According to the calculated mean H-score of SPAG6 immunoreactivity (2.31 for PV and ET; and 1.54 for PMF), patients with MPNs were divided into SPAG6 low and high expression groups. The comparisons of the clinical manifestations and laboratory features between the groups are presented in Table II. Notably, high SPAG6 expression was found to be associated with fewer splenomegaly and MF events $(\mathrm{P}<0.05)$ for all MPNs. For patients with PMF, upregulated SPAG6 expression was also found to be associated with a lower white blood cell (WBC) count $(\mathrm{P}<0.05)$, lower lactate dehydrogenase $(\mathrm{LDH})$ levels $(\mathrm{P}<0.05)$, higher hemoglobin $(\mathrm{Hb})$ levels $(\mathrm{P}<0.05)$ and an increased platelet (PLT) count $(\mathrm{P}<0.05)$. However, for 


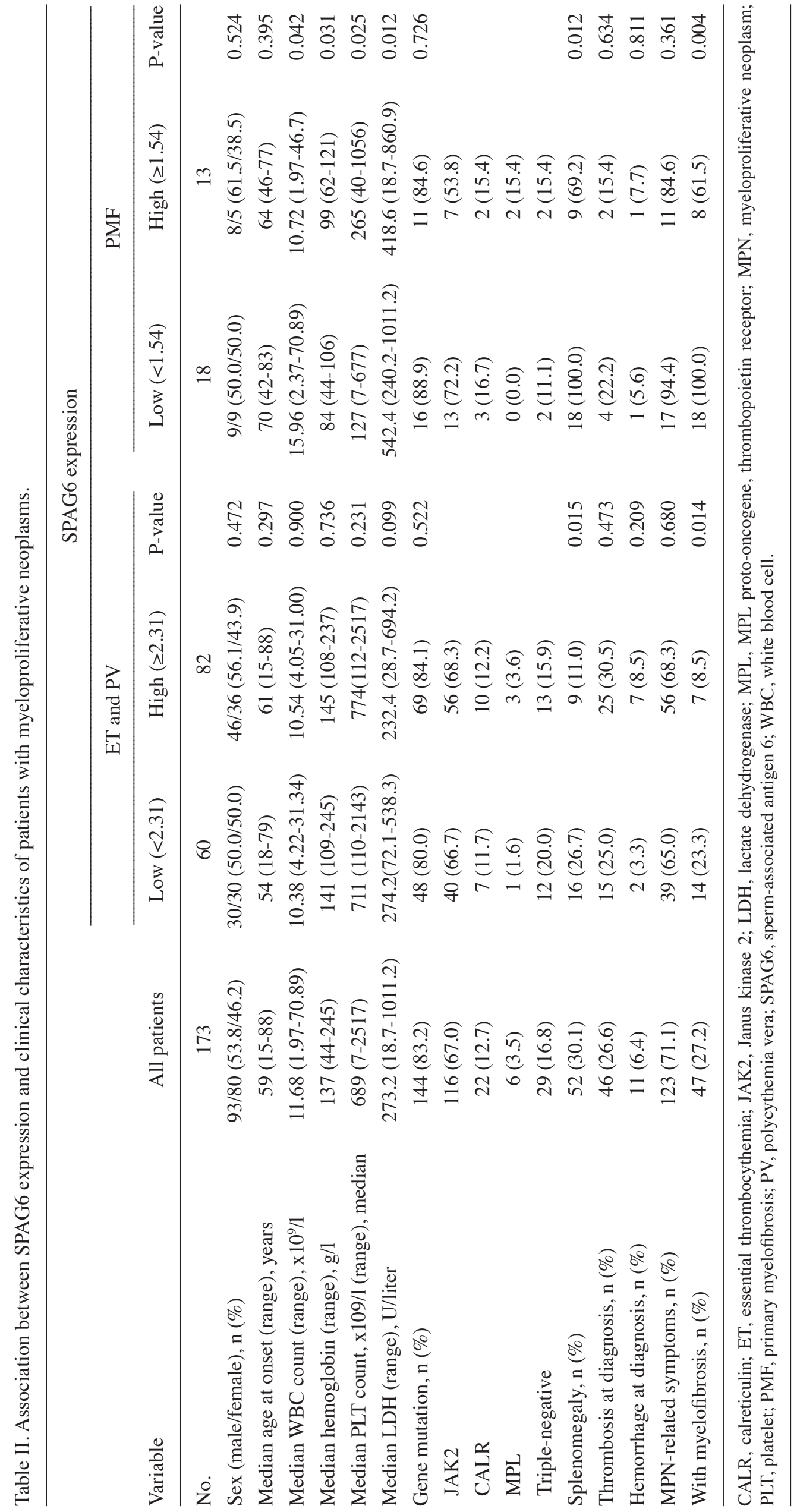



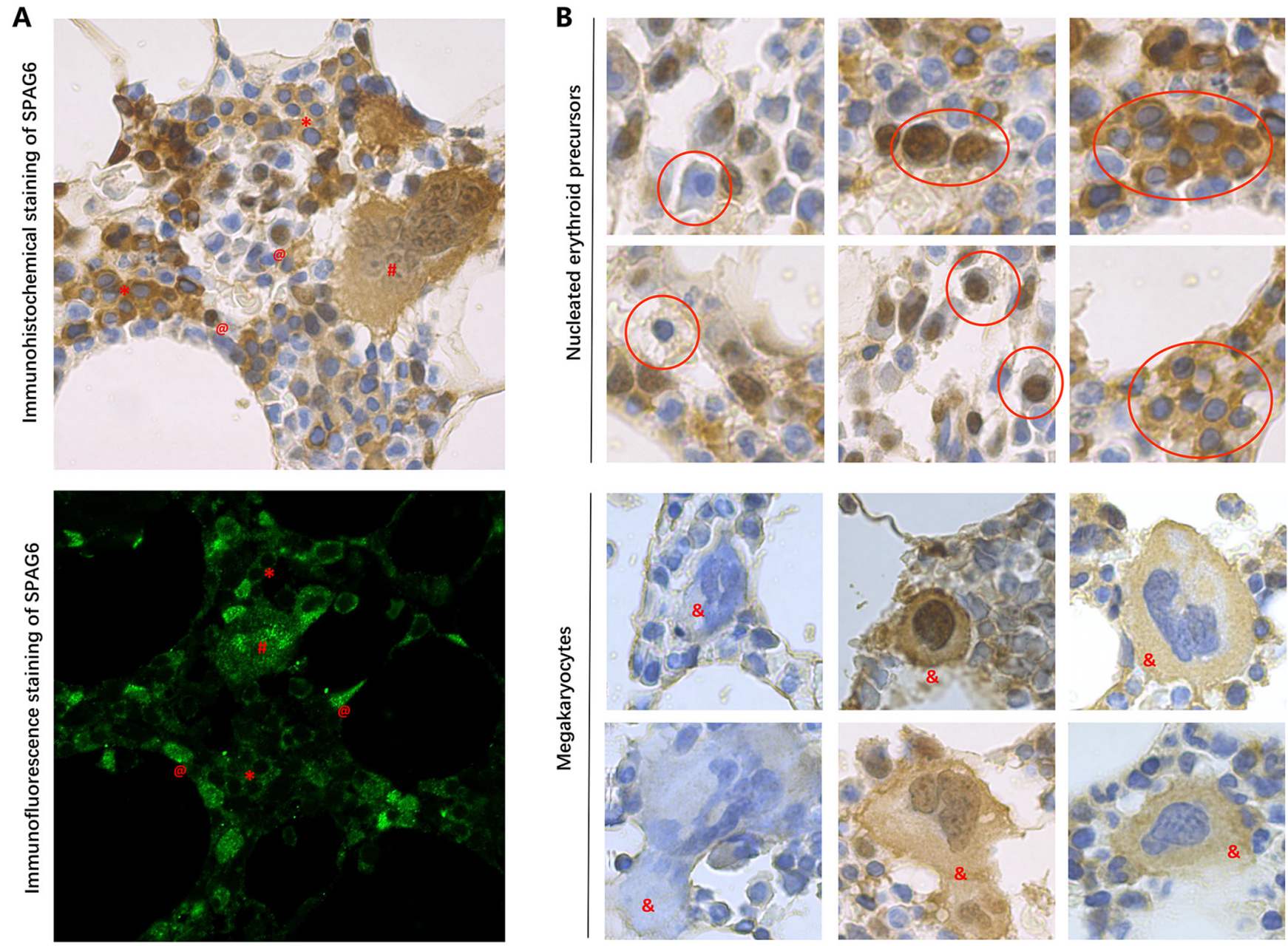

Figure 5. Subcellular localization patterns of SPAG6 protein in patients with myeloproliferative neoplasms. (A) Subcellular localization pattern of SPAG6 protein in the same patient. Immunohistochemical staining (upper panel) and immunofluorescence staining (lower panel) of SPAG6. (B) Subcellular localization pattern of SPAG6 protein in the same cell type. Nucleated erythroid precursors (upper two rows of panels; inside the red circles) and megakaryocytes (lower two rows of panels). Magnification, x1,000. @, nucleic localization; *, cytoplasmic localization; \#, nucleic and cytoplasmic localization; and \&, megakaryocyte. SPAG6, sperm-associated antigen 6.

the PV and ET group, these differences were not observed. Nevertheless, no significant differences were observed in sex, age, MPN-related symptoms, thrombosis and hemorrhage at diagnosis between the SPAG6 low and high expression groups. In addition, no significant associations between SPAG6 expression and the three driver gene mutations JAK2, CALR and MPL were found ( $\mathrm{P}>0.05)$.

Discriminative capacity of SPAG6 expression. Using the $\mathrm{H}$-score of SPAG6 immunoreactivity to differentiate patients with MPNs from healthy controls, a ROC curve was generated to examine the diagnostic value of SPAG6 expression. The results found that the AUC value was 0.9133 across all MPN cases (95\% CI, 0.8710-0.9556; P<0.0001), with a sensitivity of $81.98 \%$ and a specificity of $95.65 \%$ (Fig. 7A). Furthermore, the differentiating capacity of SPAG6 expression was also determined in patients with PV (AUC, 0.9216; 95\% CI, 0.8618-0.9815; P<0.0001; Fig. 7B), ET (AUC, 0.9278; 95\% CI, 0.8827-0.9729; P<0.0001; Fig. 7C) and PMF (AUC, 0.8569; 95\% CI, 0.7605-0.9533; P<0.0001; Fig. 7D). These results indicated that SPAG6 may be a potential biomarker for distinguishing patients with MPNs from healthy controls.

\section{Discussion}

Aberrant expression of the new cancer-testis antigen, SPAG6, has been documented in several types of neoplastic disease, such as ovarian, bladder, breast and lung cancer, and the expression levels of SPAG6 in hematological malignancies have been reported to be upregulated compared with those observed in common types of solid tumors (25). Previous studies have demonstrated that the promoter of the SPAG6 gene is methylated in breast, lung and bladder cancer, and neuroblastoma cell lines $(18,19,26,27)$. It is well established that hypermethylation of the promoter region may result in the silencing of genes. By contrast, other studies have reported that the mRNA expression levels of SPAG6 are upregulated in newly diagnosed patients with AML and MDS, while SPAG6 is expressed at low levels in healthy bone marrow samples, as well as in leukemia-free bone marrow, from patients with AML recovering from the disease after chemotherapy (14-16). To the best of our knowledge, the present study was the first to report that SPAG6 may be upregulated in MPN bone marrow cells at the mRNA and protein levels. The findings of the present study also revealed that the mRNA expression 


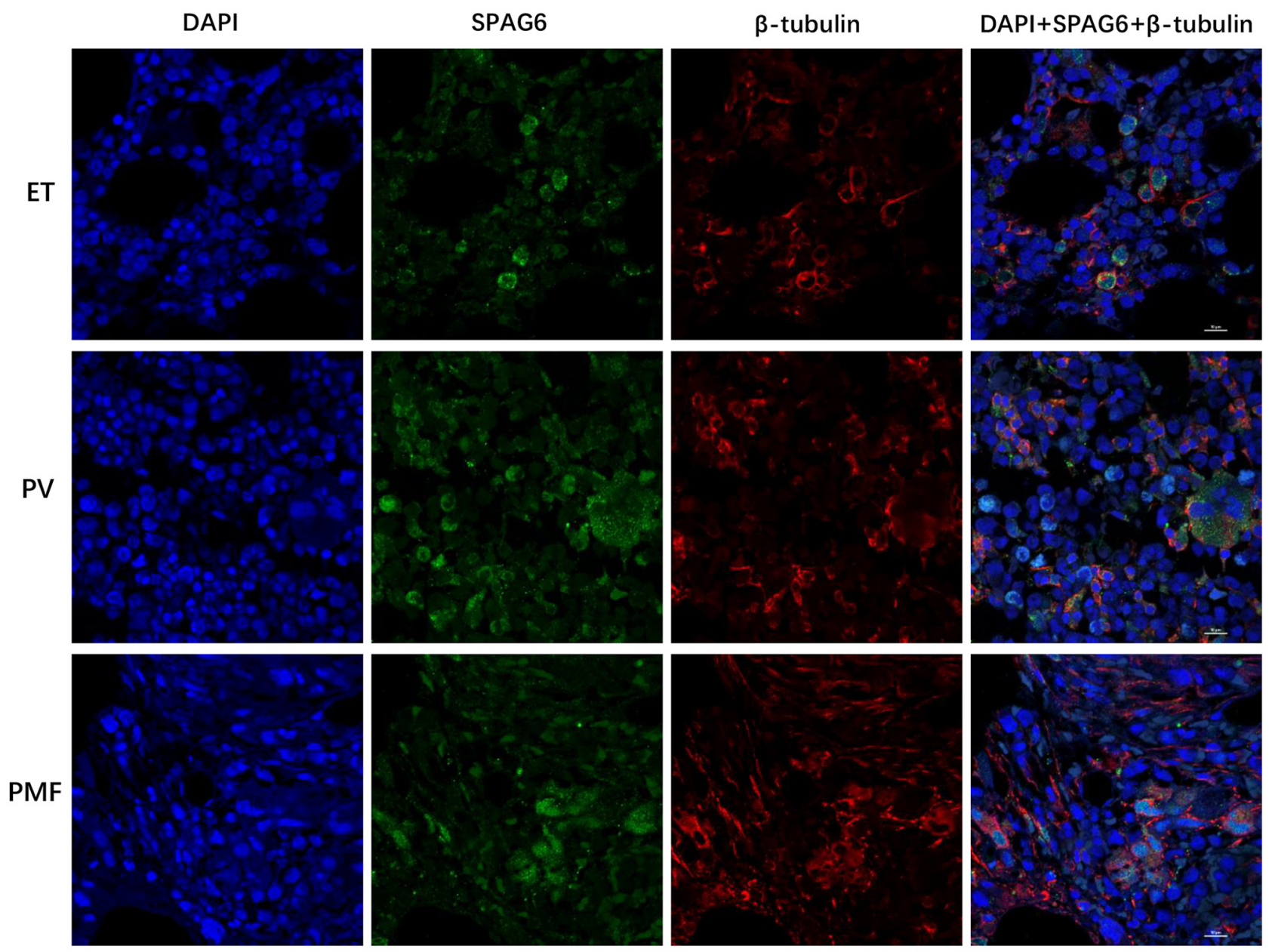

Figure 6. Double immunofluorescence staining of SPAG6 and $\beta$-tubulin expression in bone marrow biopsy specimens from patients with myeloproliferative neoplasms. ET (upper panels), PV (middle panels) and PMF (lower panels). Green fluorescence represents SPAG6 staining and red fluorescence represents $\beta$-tubulin staining. Blue staining is DAPI nuclear stain. Merged images (right panels; DAPI + SPAG6 + $\beta$-tubulin). Original magnification, $\mathrm{x} 1,000$. ET, essential thrombocythemia; PMF, primary myelofibrosis; PV, polycythemia vera; SPAG6, sperm-associated antigen 6.
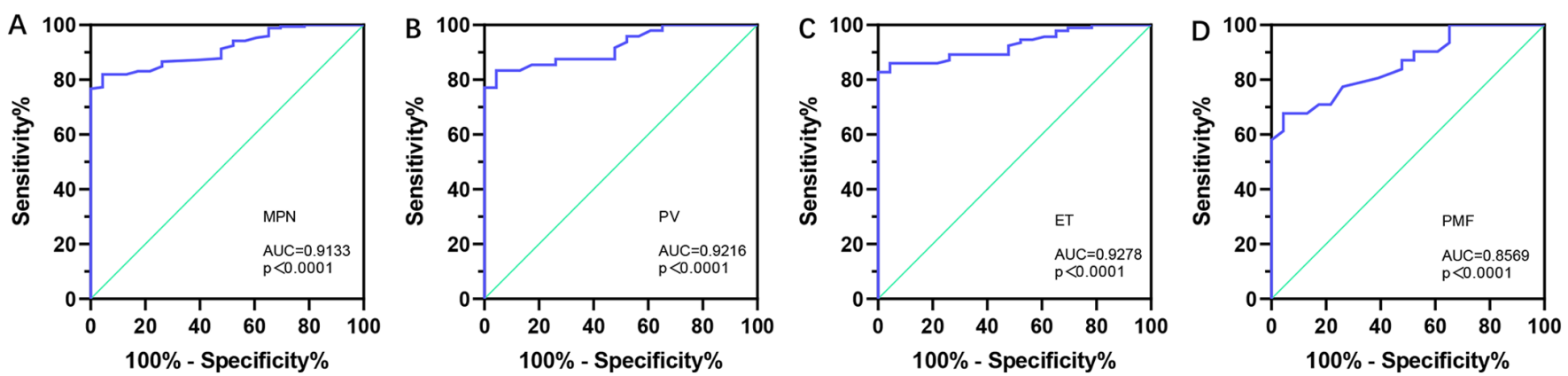

Figure 7. Receiver operating characteristic curve analysis of SPAG6 expression for distinguishing patients with MPNs from healthy controls, based on the H-score of SPAG6 immunoreactivity. (A) All patients with MPNs (95\% CI, 0.8710-0.9556; sensitivity, 81.98\%; specificity, 95.65\%). Patients with (B) PV (95\% CI, 0.8618-0.9815; sensitivity, 83.33\%; specificity, 95.65\%), (C) ET (95\% CI, 0.8827-0.9729; sensitivity, 82.80\%; specificity, 100.00\%) and (D) PMF (95\% CI, 0.7605-0.9533; sensitivity, 67.74\%; specificity, 81.43\%). AUC, area under the curve; ET, essential thrombocythemia; MPN, myeloproliferative neoplasm; PMF, primary myelofibrosis; PV, polycythemia vera; SPAG6, sperm-associated antigen 6.

levels of SPAG6 in patients with MPNs were similar to those observed in patients with MDS and AML, but were upregulated compared with those of healthy controls. These results, combined with those of previous studies (14-16), suggested that SPAG6 may be a marker of the malignant clonal expansion of bone marrow cells, and the differential expression of SPAG6 may indicate its diagnostic potential, which may prove useful for differentiating cancer-free individuals from those with hematological malignancies.

JAK2 has been identified as one of the most commonly mutated genes in the clonal hematopoiesis of MPNs $(4,7)$. Recently, it has been reported that the JAK2-V617F mutation occurs decades before a MPN diagnosis, increases the fitness of hematopoietic stem cells and induces a 
megakaryocyte/erythroid differentiation bias $(28,29)$. In addition, the JAK2-V617F mutation frequency has been reported to be higher in megakaryocyte/erythroid progenitors and lower in lymphoid and granulocyte/macrophage progenitors (28). Nearly all erythropoiesis events have been revealed to arise from the JAK2-V617F clone in PV and in most individuals diagnosed with ET (28). Notably, the results of the present study also indicated that nucleated erythroid precursors and megakaryocytes expressed high levels of SPAG6, while positive immunoreactivity of SPAG6 was only observed in individual granulocytes of 3 patients. These findings suggested that SPAG6 expression in patients with MPNs may be associated with gene mutations. Furthermore, the present study also explored whether the expression levels of SPAG6 were associated with the three main driver gene mutations. No significant associations were observed, which may be due to limited sample size.

It has been previously reported that a bias towards megakaryocyte differentiation is apparent in early multipotent stem cells in MF (29), and increased numbers of abnormal bone marrow megakaryocytes induce fibrosis, destroying the hematopoietic microenvironment and resulting in the cardinal disease features of cytopenias, namely extramedullary hematopoiesis and a high propensity for developing leukemia (30). Using immunohistochemical staining, the present study also revealed that the expression levels of SPAG6 were upregulated in patients with PMF compared with in healthy controls; however, they were downregulated compared with those observed in PV and ET cases. In addition, SPAG6 expression was downregulated in the megakaryocytes of patients with PMF. Furthermore, some of these patients $(20 / 31 ; 64.5 \%)$ with PMF were in the hematopoietic failure phase, exhibiting features of cytopenia, as the nucleated erythroid precursors number was reduced. The observed findings not only help to explain why patients with PMF had the lowest H-scores among the patients with MPN, but also suggested that aberrant SPAG6 expression may affect the MPN phenotype and participate in disease progression.

SPAG6 is mainly considered to be a microtubule-associated protein that is essential for cytoskeleton formation, and serves a role in maintaining the normal function of a variety of cells, including ciliary/flagellar biogenesis and polarization, neurogenesis, and neuronal migration $(20,31,32)$. Therefore, the present study conducted double immunofluorescence staining. The results were consistent with those observed using immunohistochemistry. The SPAG6 protein exhibited nucleic, cytoplasmic or both cytoplasmic and nucleic subcellular localization patterns in the same patient or cell type. Furthermore, it was demonstrated that the SPAG6 protein did not always co-localize with $\beta$-tubulin. These findings indicated that the SPAG6 protein may associate with multiple proteins in different cell types, or even with different proteins at different cell cycle stages in the same cell in MPNs. The SPAG6 protein contains eight consecutive WD repeats, which are known to mediate protein-protein interactions (33), and is already expressed before cilia are assembled (32). Therefore, it is not surprising that SPAG6, previously known as a motile cilia/flagella central apparatus protein, may associate with other functions in addition to participating in the regulation of the microtubule/cytoskeletal system. However, further studies are warranted to reveal the functional significance of SPAG6 upregulation and to define the molecular alterations underlying its ectopic expression and possible motile cilium-unrelated activities in MPNs.

The present study also revealed that the expression levels of SPAG6 were associated with clinical characteristics of MPNs. The association between SPAG6 expression and several clinical and laboratory characteristics was evaluated. First, the expression levels of SPAG6 were found to be associated with fewer splenomegaly and MF events. Second, the expression levels of SPAG6 in patients with PMF were associated with a lower WBC count, lower LDH levels, higher $\mathrm{Hb}$ levels and higher PLT count. Early research of patients with MPN concluded that leukocytosis is one of the risk factors for venous thrombosis, fibrotic and leukemic transformation (34). In addition, both high LDH levels and prefibrotic PMF morphology were found to be risk factors for overall survival and leukemia-free survival $(34,35)$. However, due to the long natural course (median survival, 19.8 years in ET, 13.5 years in PV and 5.9 years in PMF) of MPNs (36), the findings of the present study were insufficient to conclude whether SPAG6 expression indicates a good or poor prognosis. Therefore, investigations using a larger dataset and long-term follow-up are required to determine the disease outcomes.

While the driver mutations JAK2, CALR and MPL are found in $\sim 90 \%$ of MPN cases, there are still $\sim 10 \%$ of patients without specific molecular markers (37). Current diagnostic guidelines for triple-negative patients who do not carry the driver mutations rely on the ruling out of reactive causes of thrombocytosis and erythrocytosis combined with the examination of bone marrow biopsy specimens $(1,34)$. In the present study, the AUC value indicated that SPAG6 expression may be able to distinguish MPN cases from healthy controls, revealing that the detection of SPAG6 expression may potentially be used as a diagnostic tool to improve the accuracy of the diagnosis of MPNs.

In conclusion, to the best of our knowledge, the present study was the first to reveal that aberrant SPAG6 expression may affect the disease phenotype and serve as a tumor biomarker in BCR/ABL1-negative MPNs, although the underlying mechanism of the effect of SPAG6 in MPNs remains to be determined. Since the present study only included patients for whom sufficient material was sent to the laboratory, the study may be limited due to a possible selection bias. Therefore, the findings of the present study require further verification in follow-up studies.

\section{Acknowledgements}

Not applicable.

\section{Funding}

The present study was supported by The National Natural Sciences Foundation of China (grant no. 82070130).

\section{Availability of data and materials}

The datasets used and/or analyzed during the current study are available from the corresponding author on reasonable request. 


\section{Authors' contributions}

LD designed and performed the study, and wrote the paper. JL participated in designing the study and performed the experiments. JPZ collected clinical data and helped analyze the data. JW collected bone marrow samples and contributed to performing the experiments. ZQL, JH and LC provided technical support and participated in analyzing the data. JM contributed to the conception of the study. BZ, YRZ and LYZ collected bone marrow specimens and contributed to performing the experiments. LL conceived of and designed the research, provided financial support, and contributed to the writing and revisions of the manuscript. LD and LL confirm the authenticity of all the raw data. All authors read and approved the final manuscript.

\section{Ethics approval and consent to participate}

The use of bone marrow specimens was approved by the Ethics Committee of The First Affiliated Hospital of Chongqing Medical University (approval no. 2021-109; Chongqing, China) and written informed consent was obtained from all participants (consent was obtained from parents/guardians for minors).

\section{Patient consent for publication}

Not applicable.

\section{Competing interests}

The authors declare that they have no competing interests.

\section{References}

1. Barbui T,Thiele J, Gisslinger H, Kvasnicka HM, Vannucchi AM, Guglielmelli P, Orazi A and Tefferi A: The 2016 WHO classification and diagnostic criteria for myeloproliferative neoplasms: document summary and in-depth discussion. Blood Cancer J 8 : $15,2018$.

2. Tremblay D, Yacoub A and Hoffman R: Overview of myeloproliferative neoplasms: History, pathogenesis, diagnostic criteria, and complications. Hematol Oncol Clin North Am 35: 159-176, 2021.

3. Rampal R, Al-Shahrour F, Abdel-Wahab O, Patel JP, Brunel JP, Mermel CH, Bass AJ, Pretz J, Ahn J, Hricik T, et al: Integrated genomic analysis illustrates the central role of JAK-STAT pathway activation in myeloproliferative neoplasm pathogenesis. Blood 123: e123-e133, 2014.

4. James C, Ugo V, Le Couédic JP, Staerk J, Delhommeau F, Lacout C, Garçon L, Raslova H, Berger R, Bennaceur-Griscelli A, et al: A unique clonal JAK2 mutation leading to constitutive signalling causes polycythaemia vera. Nature 434: 1144-1148, 2005.

5. Pardanani AD, Levine RL, Lasho T, Pikman Y, Mesa RA, Wadleigh M, Steensma DP, Elliott MA, Wolanskyj AP Hogan WJ, et al: MPL515 mutations in myeloproliferative and other myeloid disorders: A study of 1182 patients. Blood 108: 3472-3476, 2006.

6. Klampfl T, Gisslinger H, Harutyunyan AS, Nivarthi H, Rumi E, Milosevic JD, Them NC, Berg T, Gisslinger B, Pietra D, et al: Somatic mutations of calreticulin in myeloproliferative neoplasms. N Engl J Med 369: 2379-2390, 2013.

7. Patel AA and Odenike O: Genomics of MPN progression. Hematology (Am Soc Hematol Educ Program) 2020: 440-449, 2020.

8. Brkic S and Meyer SC: Challenges and perspectives for therapeutic targeting of myeloproliferative neoplasms. HemaSphere 5: e516, 2020
9. Tefferi A: Primary myelofibrosis: 2021 update on diagnosis, risk-stratification and management. Am J Hematol 96: 145-162, 2021.

10. Silina K, Zayakin P, Kalniņa Z, Ivanova L, Meistere I, Endželinš E, Abols A, Stengrēvics A, Leja M, Ducena K, et al: Sperm-associated antigens as targets for cancer immunotherapy: Expression pattern and humoral immune response in cancer patients. J Immunother 34: 28-44, 2011.

11. Liu Y, Zhang L, Li W, Huang Q, Yuan S, Li Y, Liu J, Zhang S, Pin G, Song S, et al: The sperm-associated antigen 6 interactome and its role in spermatogenesis. Reproduction 158: 181-197, 2019.

12. Hu X, Yan R, Cheng X, Song L, Zhang W, Li K and Zhao S: The function of sperm-associated antigen 6 in neuronal proliferation and differentiation. J Mol Histol 47: 531-540, 2016.

13. Coan M, Rampioni Vinciguerra GL, Cesaratto L, Gardenal E, Bianchet R, Dassi E, Vecchione A, Baldassarre G, Spizzo R and Nicoloso MS: Exploring the role of fallopian ciliated cells in the pathogenesis of high-grade serous ovarian cancer. Int J Mol Sci 19: 2512, 2018.

14. Steinbach D, Bader P, Willasch A, Bartholomae S, Debatin KM, Zimmermann M, Creutzig U, Reinhardt D and Gruhn B: Prospective validation of a new method of monitoring minimal residual disease in childhood acute myelogenous leukemia. Clin Cancer Res 21: 1353-1359, 2015.

15. Mulaw MA, Krause A, Deshpande AJ, Krause LF, Rouhi A, La Starza R, Borkhardt A, Buske C, Mecucci C, Ludwig WD, et al: CALM/AF10-positive leukemias show upregulation of genes involved in chromatin assembly and DNA repair processes and of genes adjacent to the breakpoint at $10 \mathrm{p} 12$. Leukemia 26 : 1012-1019, 2012

16. Jiang M, Chen Y, Deng L, Luo X, Wang L and Liu L: Upregulation of SPAG6 in myelodysplastic syndrome: Knockdown inhibits cell proliferation via AKT/FOXO signaling pathway. DNA Cell Biol 38: 476-484, 2019.

17. Zhang R, Zhu H, Yuan Y, Wang Y and Tian Z: SPAG6 promotes cell proliferation and inhibits apoptosis through the PTEN/PI3K/ AKT pathway in Burkitt lymphoma. Oncol Rep 44: 2021-2030, 2020.

18. Mijnes J, Tiedemann J, Eschenbruch J, Gasthaus J, Bringezu S, Bauerschlag D, Maass N, Arnold N, Weimer J, Anzeneder T, et al: SNiPER: A novel hypermethylation biomarker panel for liquid biopsy based early breast cancer detection. Oncotarget 10: 6494-6508, 2019.

19. Altenberger C, Heller G, Ziegler B, Tomasich E, Marhold M, Topakian T, Müllauer L, Heffeter P, Lang G, End-Pfützenreuter A, et al: SPAG6 and L1TD1 are transcriptionally regulated by DNA methylation in non-small cell lung cancers. Mol Cancer 16: 1, 2017.

20. Zheng DF, Wang Q, Wang JP, Bao ZQ, Wu SW, Ma L, Chai DM, Wang ZP and Tao YS: The emerging role of sperm-associated antigen 6 gene in the microtubule function of cells and cancer. Mol Ther Oncolytics 15: 101-107, 2019.

21. Li X, Xu L, Sun G, Wu X, Bai X, Li J, Strauss JF, Zhang Z and Wang H: Spag6 mutant mice have defects in development and function of spiral ganglion neurons, apoptosis, and higher sensitivity to paclitaxel. Sci Rep 7: 8638, 2017.

22. Zhang M, Luo J, Luo X and Liu L: SPAG6 silencing induces autophagic cell death in SKM-1 cells via the AMPK/mTOR/ ULK1 signaling pathway. Oncol Lett 20: 551-560, 2020.

23. Livak KJ and Schmittgen TD: Analysis of relative gene expression data using real-time quantitative PCR and the 2(-Delta Delta $\mathrm{C}(\mathrm{T})$ ) method. Methods 25: 402-408, 2001.

24. Yang Y, Xiao M, Song Y, Tang Y, Luo T, Yang S, He W, Cheng Q, $\mathrm{Ma} \mathrm{L}$, Zhang $\mathrm{Y}$, et al: $\mathrm{H}$-score of $11 \beta$-hydroxylase and aldosterone synthase in the histopathological diagnosis of adrenocortical tumors. Endocrine 65: 683-691, 2019.

25. Barretina J, Caponigro G, Stransky N, Venkatesan K, Margolin AA, Kim S, Wilson CJ, Lehár J, Kryukov GV, Sonkin D, et al: The Cancer Cell Line Encyclopedia enables predictive modelling of anticancer drug sensitivity. Nature 483: 603-607, 2012.

26. Kitchen MO, Bryan RT, Haworth KE, Emes RD, Luscombe C, Gommersall L, Cheng KK, Zeegers MP, James ND, Devall AJ, et al: Methylation of HOXA9 and ISL1 predicts patient outcome in high-grade non-invasive bladder cancer. PLoS One 10: e0137003, 2015.

27. Abe M, Watanabe N, McDonell N, Takato T, Ohira M, Nakagawara A and Ushijima T: Identification of genes targeted by $\mathrm{CpG}$ island methylator phenotype in neuroblastomas, and their possible integrative involvement in poor prognosis. Oncology 74: 50-60, 2008. 
28. Van Egeren D, Escabi J, Nguyen M, Liu S, Reilly CR, Patel S, Kamaz B, Kalyva M, DeAngelo DJ, Galinsky I, et al: Reconstructing the lineage histories and differentiation trajectories of individual cancer cells in myeloproliferative neoplasms. Cell Stem Cell 28: 514-523.e9, 2021.

29. Psaila B, Wang G, Rodriguez-Meira A, Li R, Heuston EF, Murphy L, Yee D, Hitchcock IS, Sousos N, O'Sullivan J, et al; NIH Intramural Sequencing Center: Single-cell analyses reveal megakaryocyte-biased hematopoiesis in myelofibrosis and identify mutant clone-specific targets. Mol Cell 78: 477-492.e8, 2020.

30. O'Sullivan JM and Harrison CN: Myelofibrosis: Clinicopathologic features, prognosis, and management. Clin Adv Hematol Oncol 16: 121-131, 2018.

31. Cooley LF, El Shikh ME, Li W, Keim RC, Zhang Z, Strauss JF, Zhang $\mathrm{Z}$ and Conrad DH: Impaired immunological synapse in sperm associated antigen 6 (SPAG6) deficient mice. Sci Rep 6: $25840,2016$.

32. Li W, Mukherjee A, Wu J, Zhang L, Teves ME, Li H, Nambiar S, Henderson SC, Horwitz AR, Strauss JF III, et al: Sperm associated antigen 6 (SPAG6) regulates fibroblast cell growth, morphology, migration and ciliogenesis. Sci Rep 5: 16506, 2015.

33. Tewari R, Bailes E, Bunting KA and Coates JC: Armadillo-repeat protein functions: Questions for little creatures. Trends Cell Biol 20: 470-481, 2010.
34. Tefferi A and Barbui T: Polycythemia vera and essential thrombocythemia: 2019 update on diagnosis, risk-stratification and management. Am J Hematol 94: 133-143, 2019.

35. Carobbio A, Guglielmelli P, Rumi E, Cavalloni C, De Stefano V, Betti S, Rambaldi A, Finazzi MC, Thiele J, Vannucchi AM, et al: A multistate model of survival prediction and event monitoring in prefibrotic myelofibrosis. Blood Cancer J 10: 100, 2020.

36. Tefferi A, Guglielmelli P, Larson DR, Finke C, Wassie EA, Pieri L, Gangat N, Fjerza R, Belachew AA, Lasho TL, et al: Long-term survival and blast transformation in molecularly annotated essential thrombocythemia, polycythemia vera, and myelofibrosis. Blood 124: 2507-2513, quiz 2615, 2014.

37. Tefferi A, Lasho TL, Finke CM, Knudson RA, Ketterling R, Hanson CH, Maffioli M, Caramazza D, Passamonti F and Pardanani A: CALR vs. JAK2 vs MPL-mutated or triple-negative myelofibrosis: Clinical, cytogenetic and molecular comparisons. Leukemia 28: 1472-1477, 2014.

This work is licensed under a Creative Commons Attribution-NonCommercial-NoDerivatives 4.0 International (CC BY-NC-ND 4.0) License. 